\title{
A model for the analysis of the heat release from a supercooled liquid at the glass transition
}

\author{
M RAJESWARI ${ }^{\dagger}$ and A K RAYCHAUDHURI \\ Department of Physics, Indian Institute of Science, Bangalore 560012, India \\ ${ }^{\dagger}$ Present address: Center for Superconductivity Research, Department of Physics, University \\ of Maryland, College Park, MD 20742, USA
}

MS received 19 April 1993; revised 17 September 1993

\begin{abstract}
In this paper we present a phenomenological model to analyze the heat release at the glass transition as observed in the continuous cooling calorimetry when a supercooled liquid freezes into the glassy state. We developed this model for the quantitative analysis of the experimental data to obtain the specific heat and the parameters which govern the structural relaxation. A description of the model and the detailed analysis are presented and the relaxation parameters are compared with the corresponding values obtained from the specific heat spectroscopy. Our analysis reveals several interesting aspects which include the effects of delayed enthalpy relaxation and the nonequilibrium. structural relaxation time on the observed specific heat, the temperature dependence of the equilibrium configurational specific heat and the validity of the Vogel-Fulcher equation for the relaxation time.
\end{abstract}

Keywords. Time-dependent specific heat; glass transition; structural relaxation.

\section{PACS Nos 64.70; 65.4}

\section{Introduction}

The experimentally observed specific heat undergoes a step-like fall in the temperature range where the supercooled liquid freezes into the glassy state. This fall in the specific heat near the glass transition temperature $\left(T_{\mathrm{g}}\right)$ is largely associated with the increase in the structural relaxation time $\tau_{\mathrm{s}}$ with decreasing temperature. In the glass transition temperature range where $\tau_{\text {s }}$ becomes comparable to the experimental time scale $\tau_{\exp }$, the configurational degrees of freedom do not contribute to the observed changes in enthalpy. This accounts for the fall in the specific heat. The observed specific heat at the glass transition is a nonequilibrium time dependent quantity which contains information about the nature of the structural relaxation. Recently we have reported our studies of the specific heat at the glass transition which employ a novel technique known as the continuous cooling calorimetry $[1,2]$. In this paper we describe a phenomenological model which we have developed to enable us to obtain the parameters governing the structural relaxation from the observed heat release at the glass transition. Since the specific heat at the glass transition is a nonequilibrium quantity, the measured specific heat is sensitive to the kinetics of the calorimetric technique employed.

At the outset we would like to point out that the continuous cooling calorimetry at the glass transition is distinct from most of the earlier studies of the glass transition 


\section{$M$ Rajeswari and $A K$ Raychaudhuri}

which employ differential scanning calorimetry (DSC) [3] or adiabatic calorimetry $[4,5]$ where the specific heat measured as a quenched glass is reheated through the glass transition range. The specific heat observed during this reheating is strongly influenced by the relaxation of the enthalpy frozen-in during quenching. The specific heat peaks observed at the glass transition in the heating experiments (as in DSC and adiabatic calorimetry) have their origin in the delayed enthalpy relaxation. The analysis and modeling of these experiments [3] are thus complicated by the thermal history built into the system during quenching. In contrast, in the continuous cooling method, the specific heat is measured as the supercooled liquid freezes into the glassy state from an initial equilibrium state at $T \gg T_{\mathrm{g}}$. In this case there is no thermal history built into the system prior to the cooling process. The specific heat during cooling does not show the enthalpy relaxation peaks seen in the data during reheating after quenching. As a result, the modeling and the analysis are simpler and more direct here. The continuous cooling calorimetry may be thought of as a large amplitude time domain analog of the specific heat spectroscopy experiment [6] which studies the specific heat in the linear response regime in the supercooled liquid. It should be mentioned here that when the glass is thermally cycled through $T_{\mathrm{g}}$ the specific heat shows a dip below $T_{\mathrm{g}}$ which is sensitive to the thermal history [1]. However in the present report we do not discuss the thermal cycling experiments.

In the remainder of this paper we describe the development of the model and show its application to the quantitative analysis of the data in one glass former (glycerol). The model is general and may be applied to any glass former. It may also be extended for the analysis of similar data (other than specific heat) where one deals with kinetic freezing.

In $\S 2$ we briefly describe the experiment and present some typical data. In $\S 3$ we discuss our treatment of the time dependent specific heat and the structural relaxation time. In $\S 4$ we present the model followed by its application to glycerol in $\S 5$. In $\S 6$ we discuss the structural relaxation parameters obtained for glycerol which is followed by the concluding summary in $\S 7$.

\section{The experimental technique}

The continuous cooling method of specific heat measurement which is based on the principles of relaxation calorimetry [7] has been described in detail earlier [2]. The sample kept in a vacuum environment is linked to a heat bath maintained at liquid nitrogen temperature by a thermal link whose heat loss rate $\dot{Q}(T)$ is experimentally determined. The sample is annealed at a temperature $T_{\mathrm{A}}>T_{\mathrm{g}}$ by holding it at $T_{\mathrm{A}}$ for several hours. The heat input is then turned off and the sample cools below $T_{\mathrm{g}}$ by losing heat through the link. The specific heat is determined from the cooling rate $\mathrm{d} T / \mathrm{d} t$ as the sample cools from $T_{\mathrm{A}}$. The cooling process is shown schematically in figure 1 . For glycerol the values of $T_{\mathrm{A}}, T_{\mathrm{g}}$ and the average cooling rate are also indicated in the figure.

In the absence of any heat input, the heat capacity of the cooling thermal mass is related to the cooling rate $\mathrm{d} T / \mathrm{d} t$ by

$$
C(T)=\dot{Q}(T) /(\mathrm{d} T / \mathrm{d} t)
$$

Thus the heat capacity may be obtained if the cooling rate $d T / d t$ and the heat loss rate through the link $\dot{Q}(T)$ are known. In this experiment we measure $T(t)$ as the 
A model for the analysis of the heat release

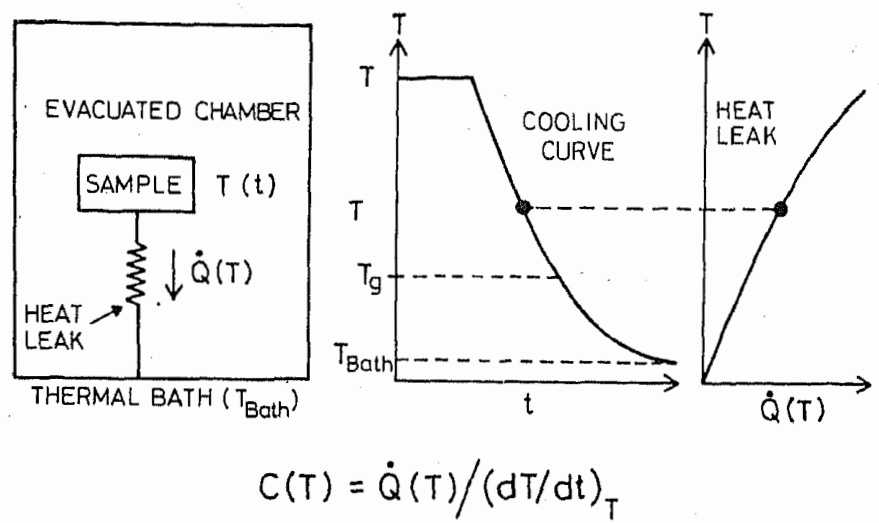

For Glycerol $T_{A} \approx 230 \mathrm{~K}, T_{\text {Bath }}=77 \mathrm{~K}, T_{g}=185 \mathrm{~K}$

Average cooling rate $=.03 \mathrm{~K} / \mathrm{s}$

Figure 1. Schematics of the experimental set-up along with the measured cooling curve $(T-t)$ and the heat leak curve $(Q(T)-T)$. The slope $(\mathrm{d} T / \mathrm{d} t)_{T}$ is obtained from the cooling curve. $Q(T)$ is determined in a separate run as described in the text.

sample cools through the link from which the cooling rate $d T / d t$ is determined. The rate of heat loss through the link $\dot{Q}(T)$ is determined separately by measuring the power input needed to stabilize the sample at several closely spaced temperature values and subsequently obtaining a polynomial fit. $\dot{Q}(T)$ is then a measure of the heat release from the sample which arises from the enthalpy relaxation in a time scale faster than the experimental time scale $\tau_{\exp }$ corresponding to the cooling rate employed. Equation (1) strictly holds in the temperature regime where the structural relaxation time $\tau_{\mathrm{s}}$ satisfies the condition $\tau_{\mathrm{s}} \ll \tau_{\mathrm{exp}}$. In the glass transition region where $\tau_{\mathrm{s}} \sim \tau_{\exp }$ and in the sub- $T_{\mathrm{g}}$ region where $\tau_{\mathrm{s}} \gg \tau_{\exp }, C(T)$ will not be the complete equilibrium heat capacity as the configurational states do not equilibrate in the time scale $\tau_{\exp }$. We can however treat (1) as an operational definition of the heat capacity. In the analysis that follows, we show how we relate the observed heat capacity to the equilibrium heat capacity in the framework of a kinetic model of enthalpy relaxation.

In figure 2 we present the specific heat data of glycerol and compare it with the data from earlier adiabatic calorimetric experiments [8]. The data and analysis for other glass formers we have studied may be found elsewhere [9]. (Here we focus on glycerol as a representative case of our analysis). As seen in figure 2, in the supercooled liquid region and the sub- $T_{\mathrm{g}}$ region there is reasonable agreement (to within $\sim 5 \%$ ) between the two sets of data. The glass transition region however shows significant variation which is to be expected since the data in this range are sensitive to the experimental kinetics. In table 1 we compare the different parameters associated with the glass transition namely the glass transition temperature $T_{\mathrm{g}}$, the width of the transition $\Delta T_{\mathrm{g}}$ and the change in the specific heat across the transition $\Delta C_{p}$. In earlier studies these quantities have been defined by employing various arbitrary criteria. We define these quantities from the derivatives of the $C(T)$ curves which allows a less arbitrary definition. Since we obtain $C(T)$ in a continuous temperature sweep, the data are sufficiently closely-spaced in temperature to determine the derivatives $\mathrm{d} C / \mathrm{d} T$ and $\mathrm{d}^{2} C / \mathrm{d} T^{2}$ with sufficient accuracy. The change in the specific heat across 
$M$ Rajeswari and $A K$ Raychaudhuri

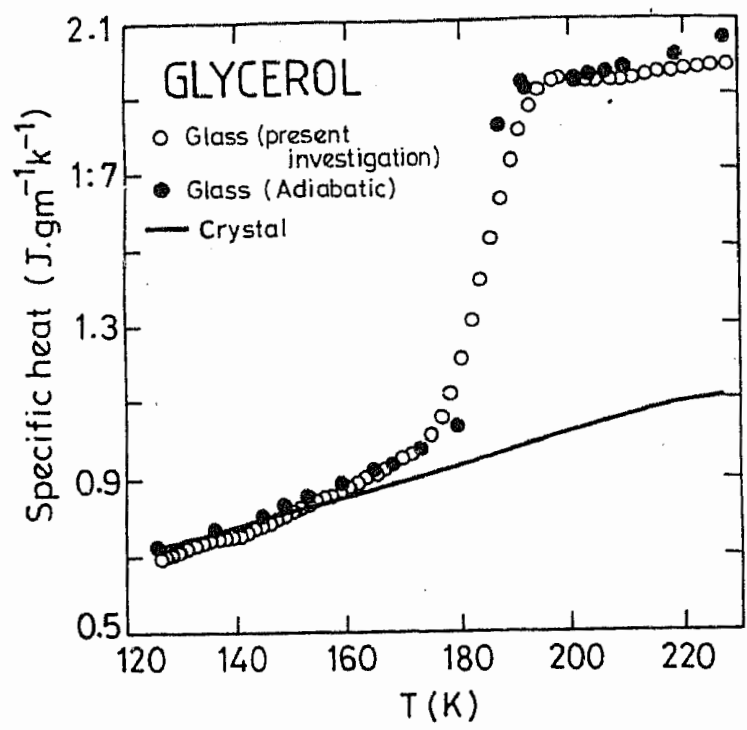

Figure 2. The specific heat of glycerol obtained from continuous cooling calorimetry compared to the values reported in literature [9].

Table 1. The glass transition temperature $\left(T_{\mathrm{g}}\right)$, transition width $\Delta T_{\mathrm{g}}$ and the specific heat change across the transition $\left(\Delta C_{p}\right)$ of glycerol from the continuous cooling calorimetry compared with the same quantities from adiabatic calorimetry.

\begin{tabular}{lcc}
\hline & $\begin{array}{c}\text { Continuous cooling } \\
\text { calorimetry } \\
\text { (present study) }\end{array}$ & Adiabatic calorimetry [8] \\
\hline$T_{\mathrm{g}}(\mathrm{K})$ & 185 & $180-190$ \\
$\Delta T_{\mathrm{g}}(\mathrm{K})$ & 29 & 19 \\
$\Delta C_{\mathrm{p}}$ & 0.97 & 0.91 \\
$\left(\mathrm{Jg}^{-1} \mathrm{~K}^{-1}\right.$ ) & 0.50 & 0.47 \\
$\Delta C_{\mathrm{p}} / C_{\mathrm{p}}$ (liquid) & 0.50 & 0 \\
\hline
\end{tabular}

the glass transition interval is defined as

$$
\Delta C_{p}=C_{p}\left(T_{u}\right)-C_{p}\left(T_{l}\right)
$$

where $T_{u}$ and $T_{l}$ correspond to the upper and lower limits of the transition interval as indicated in figure 3. The width of the transition interval is defined as $\Delta T_{g}=T_{u}-T_{l}$. As shown in table 1 the quantities $T_{\mathrm{g}}$ and $\Delta T_{\mathrm{g}}$ as obtained from the continuous cooling calorimetry experiment differ from the adiabatic calorimetry data, the difference being due to the variation in $\tau_{\text {exp }}$.

Our task in the following sections is to obtain quantitative information about the structural relaxation from the $C(T)$ data in figure 3 .

3. The time-dependent specific heat and structural relaxation time near the glass transition

A glass being a system frozen far from equilibrium, the specific heat is time dependent at all $T<T_{\mathrm{g}}$. However there are only two temperature regions where the time 
A model for the analysis of the heat release

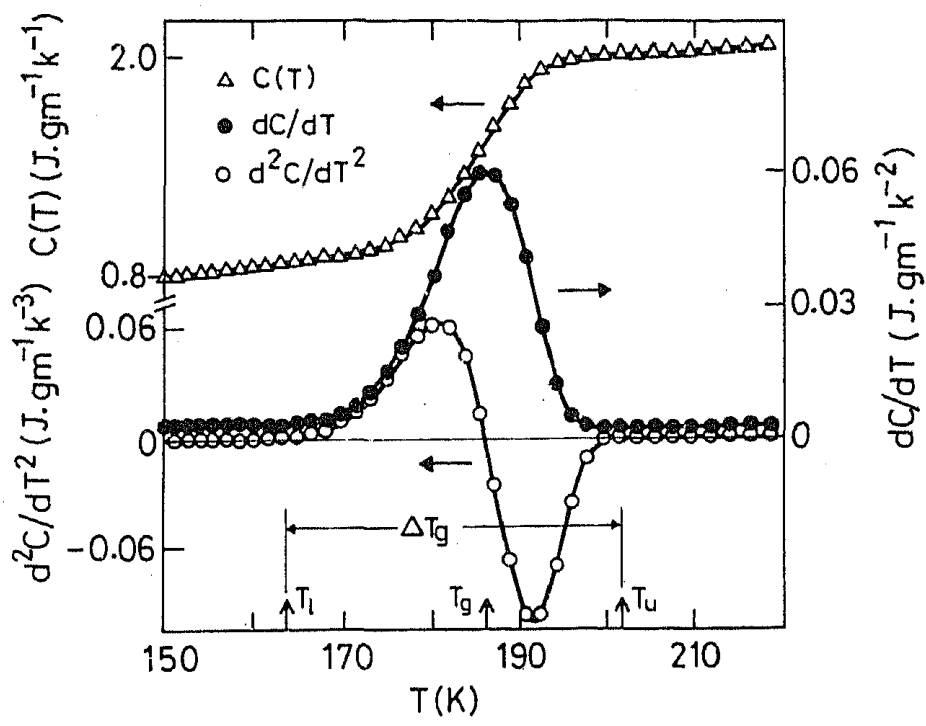

Figure 3. The specific heat $C(T)\left(\mathrm{Jg}^{-1} \mathrm{~K}^{-1}\right)$ and its temperature derivatives $\mathrm{d} C / \mathrm{d} T\left(\mathrm{Jg}^{-1} \mathrm{~K}^{-2}\right)$ and $\mathrm{d}^{2} C / \mathrm{dT}^{2}\left(\mathrm{Jg}^{-1} \mathrm{~K}^{-3}\right)$ of glycerol indicating the glass transition interval $\left(T_{\mathrm{g}}\right.$ and $\left.\Delta T_{\mathrm{g}}\right)$.

dependence is experimentally observable. First is the region at $T \sim T_{\mathrm{g}}$ where the enthalpy change associated with the structural relaxation is observable in experimental time scales. The second is another temperature range at very low temperatures $(T<1 \mathrm{~K})$ where the time dependent specific heat associated with the tunneling states is observable.

The heat capacity of the system in the supercooled state and the glass transition region may be considered to be made up of two components - the lattice component $C_{L}$ and the configurational component $C_{\mathrm{F}}$. The lattice component includes contribution to the heat capacity from the vibrational degrees of freedom in the sample which equilibrate in time scales $\sim 10^{-12} \mathrm{~s}$ which is much shorter than the time scale of observation. $C_{L}$ could also include contributions from configurational degrees (such as localized motion about defect sites) with short relaxation time $\left(\tau_{s} \ll \tau_{\text {exp }}\right)$. The second component of the heat capacity denoted as $C_{\mathrm{F}}$ is associated with the configurational degrees of freedom which relax on time scales resolvable by experiment (i.e. $\tau_{s} \sim \tau_{\text {exp }}$ ) in the glass transition range. (It should be mentioned here that considering the glass heat capacity to be the sum of two components is only an operational approximation and is not rigorously valid). The relaxational nature of $C_{\mathrm{F}}$ is then manifested in the experimental observation as a time dependent specific heat. In the framework of the relaxational models of the glass transition the time dependence of $C_{F}$ may be expressed as

$$
C_{\mathbf{F}}(T, t)=C_{\mathrm{Fo}}[1-\phi(T, t)] \text {. }
$$

Here $C_{\mathrm{FO}}$ is the complete equilibrium heat capacity which is observable only in the temperature range where $\tau_{\mathrm{s}} \ll \tau_{\exp }$ and $\phi(T, t)$ is the relaxation function describing the time dependence of the configurational enthalpy change. The relaxation function at a temperature $T$ may be defined from the enthalpy change corresponding to an instantaneous change in temperature $\Delta T$ as

$$
\phi(T, t)=\frac{H(T, t)-H_{\mathrm{eq}}(T)}{H\left(T, t=0^{+}\right)-H_{\mathrm{eq}}(T)} .
$$


At $t \sim 0 \phi(T, t)=1$ and at $t \rightarrow \infty H(T, t)=H_{\mathrm{eq}}(T)$ and $\phi(T, t)=0$. The significant change of $\phi(T, t)$ occurs in the time scale $\sim \tau_{\mathrm{s}}$.

The relaxation function associated with the supercooled liquid and glassy relaxations have been found to be nonlinear and non-debye in nature. The non-debye nature is observed in response to even infinitesimal perturbations from equilibrium while nonlinearity arises in the regime of large amplitude perturbations. An empirical expression which has been found to describe a variety of non-debye relaxation processes [10] is of the form

$$
\phi(T, t)=\exp \left[-\left(t / \tau_{\mathrm{s}}(T, t)\right)^{\beta}\right]
$$

here $\tau_{\mathrm{s}}$ is the structural relaxation time and $\beta$ is a parameter $(0<\beta<1)$ related to the width of the spectrum of relaxation times. The limit $\beta=1$ corresponds to the Debye relaxation with a single relaxation time. We have used the form of $\phi(T, t)$ in (3) in our model of the enthalpy relaxation.

The temperature dependence of the structural relaxation time $\tau_{\mathrm{s}}$ and its nonequilibrium nature primarily govern the relaxation at the glass transition. In addition to the strong temperature dependence, $\tau_{\mathrm{s}}$ is also time dependent near the glass transition because the structure itself relaxes. Thus the effective value of $\tau_{\mathrm{s}}$ is different from its value $\tau_{\text {eq }}(T)$ associated with the equilibrium structure at a temperature $T$. We will discuss the time dependence of $\tau_{\mathrm{s}}$ shortly. The temperature dependence of $\tau_{\text {eq }}$ has been found to obey the empirical relation

$$
\tau_{\text {eq }}=\tau_{0} \exp \left[A /\left(T-T_{0}\right)\right]
$$

which is referred to as the Vogel-Tamman-Fulcher (VTF) expression. The VTF expression (as distinct from the Arrhenius form with $T_{0}=0$ ) implies that the apparent activation energy increases with decreasing temperature and $\tau_{\text {eq }}$ diverges at a finite temperature $T_{0}$. We have used the VTF form for the temperature dependence of $\tau_{\text {eq }}$ in our model. The parameter $T_{0}$ is usually taken to be temperature independent and a thermodynamic significance is associated with it as representing an ideal phase transition underlying the kinetic glass transition [11]. We find that we need to incorporate a temperature dependent $T_{0}$ in our model to adequately describe the experimental observations. We discuss this aspect in a later section:

As already mentioned, the relaxation function $\phi(T, t)$ near the glass transition exhibits nonlinear behavior in response to large amplitude perturbations. The nonlinear behavior is associated with the nonequilibrium nature of the relaxation time $\tau_{\mathrm{s}}$ which, as already discussed, has a time dependence in addition to the temperature dependence. The time dependent $\tau_{s}$ attains its equilibrium value $\tau_{\text {eq }}(T)$ only when the system is retained isothermally at $T$ for a time longer than $\tau_{\text {eq }}$ itself. Thus in (4) we need to incorporate the time dependence of the relaxation time. The time dependence arises due to the dependence of the relaxation time on the structural state which itself is changing during the course of the relaxation process. The dependence of the nonequilibrium relaxation time on structure implies that if the system is not in its equilibrium state, $\tau_{\mathrm{s}}$. would depend on thermal history and hence is not a unique function of temperature. The nonequilibrium nature of $\tau_{\mathrm{s}}$ becomes significant for large amplitude perturbations from equilibrium as in the present experiment.

The time dependent relaxation time has been described by a phenomenological model [12] which uses the fictive temperature concept [13] to quantify the structural 
state of the system. The relaxation time $\tau_{\mathrm{s}}$ is considered to depend on the temperature $T$ as well as on the fictive temperature $T_{\mathrm{F}}$.

Another equivalent approach has been developed by Kovacs [14] to take into account the time dependence of $\tau_{s}$. Kovacs's method considers the nonequilibrium relaxation time as a function of the temperature as well as of the excess enthalpy or the excess free volume in the system. The excess enthalpy or the excess free volume is a measure of the deviation of the system from equilibrium. The nonequilibrium nature of $\tau_{s}$ is thus taken into account by its dependence on the structural state through a quantity which tracks the deviation of the system from the equilibrium configuration. We have used Kovacs' model to incorporate the time dependence of $\tau_{\mathrm{s}}$ in our model. Our choice of this model is based on the computational ease. Kovacs' approach should be essentially equivalent to Narayanaswamy's model since both are one parameter models which take into account the time dependence of the relaxation time. We will show the equivalence of the two approaches later on. In a separate section, we describe in detail Kovacs' approach to calculate the time dependent $\tau_{s}$.

\section{Description of the model}

We now present the model which describes the heat release and the specific heat observed in the continuous cooling experiment as the supercooled liquid is cooled through the glass transition interval starting from a temperature $T>T_{\mathrm{g}}$. The essential feature of this model is that it keeps track of: (i) the heat released from the system during a cooling step, (ii) the heat trapped due to the incomplete relaxation and (iii) the delayed heat release in subsequent steps. Figure 4 is a schematic representation of the model. The heat capacity of the thermal mass connected to the heat bath (via. the thermal link) is made up of two components - the lattice component $C_{L}$ and the configurational component $C_{F}$. $C_{L}$ is associated with an instantaneous response in the time scale of the experiment while $C_{F}$ has an observable time dependence given by (2) and (3). As shown in figure 4, there is an effective heat source $Q^{h}$ present in the system which arises due to the delayed heat release from the configurational modes as the system continuously moves towards thermal equilibrium. The delayed heat release is associated with the unrelaxed enthalpy in the system which we call the trapped heat the nature of which we discuss shortly.

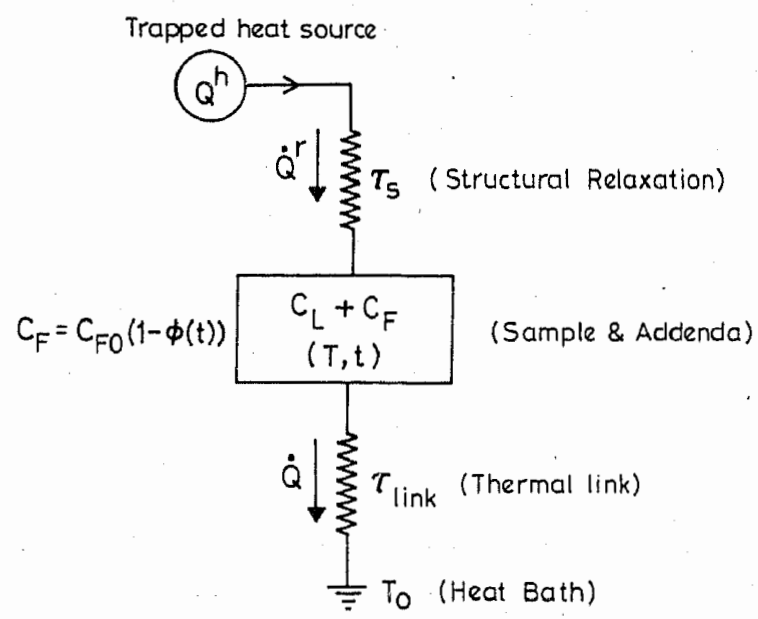

Figure 4. Schematic representation of the model. 


\section{$M$ Rajeswari and $A K$ Raychaudhuri}

We consider the system comprising of $C_{\mathrm{L}}+C_{\mathrm{F}}$ cooling through the link from a temperature $T>T_{\mathrm{g}}$. The continuous cooling process in the experiment is approximated by a step cooling program in the model. The cooling occurs in temperature steps of $\Delta T$ followed by isothermal holds of duration $\Delta t$ with

$$
\Delta t=\Delta T / q
$$

where $q$ is a cooling rate appropriate to the experiment. It may be mentioned here that the relaxational behavior observed in our model is not sensitive to the particular value of $\Delta t$ chosen as long as $\Delta T / \Delta t$ corresponds to the cooling rate $q$ and the temperature step $\Delta T$ is not too large. We have taken a value of $\Delta t=60 \mathrm{~s}$, the corresponding $\Delta T$ at $T_{\mathrm{g}}$ being $\sim 1 \mathrm{~K}$. This choice was mainly decided by considerations of the computation time.

One of the observed quantities in our experiment is the cooling rate $(\mathrm{d} T / \mathrm{d} t)$ from which the specific heat is calculated using (1). Analogously, in the model we compute the cooling rate of the system (consisting of the sample plus the addenda) the heat capacity of which is given by $C_{\mathrm{L}}+C_{\mathrm{F}}$. We denote the effective time dependent heat capacity of the system as $C_{\text {eff }}(T, t)$ given by

$$
C_{\text {eff }}(T, t)=C_{\mathrm{L}}(T)+C_{\mathrm{FO}}(T)[1-\phi(T, t)]
$$

[Note that the second term in this equation is the time-dependent specific heat in (2)].

In the above equation and the subsequent discussions, the time interval $t$ is the isothermal hold time $\Delta t$. At $t \ll \tau_{s}$ (i.e. $t=0^{+}$) it can be seen from equation 2 (a) that $\phi(T, t) \sim 1$ and $C_{\text {eff }}(T, t) \sim C_{\mathrm{L}}$. At longer times $t \gg \tau_{\mathrm{s}}, \phi(T, t) \sim 0$ and $C_{\text {eff }}(T, t) \sim C_{\mathrm{L}}(T)+$ $C_{\mathrm{FO}}(T)$. Since $\tau_{\mathrm{s}}$ is a rapidly varying function of temperature, cooling the liquid through $T_{\mathrm{g}}$ is equivalent to going from the limit $t \gg \tau_{\mathrm{s}}$ (at $T>T_{\mathrm{g}}$ ) to $t \ll \tau_{\mathrm{s}}\left(\right.$ at $T<T_{\mathrm{g}}$ ) and $C_{\text {eff }}(T, t)$ in effect gives the relaxation function $\phi(T, t)$. If the thermal link transports heat at a rate $\dot{Q}(T)$ as a result of which the temperature of the system changes by $\Delta T$ in a time interval $\Delta t$ we have the following heat balance equation

$$
\Delta T=\left(\dot{Q}(T) \Delta t-Q_{\mathrm{n}}^{r}\right) / C_{\text {eff }}(T, t) .
$$

In the above equation the signs of the terms are so chosen that the cooling process represents a positive $\Delta T$. The term $Q_{\mathrm{n}}^{r}$ in the numerator arises from the effective heat source $Q^{h}$ shown in figure 4 which is due to the delayed heat release associated with the unrelaxed excess enthalpy. The excess enthalpy which cannot relax in a given time step relaxes progressively during subsequent steps giving rise to the delayed heat release from the system. We call this the trapped heat release $Q^{r}$. We calculate the trapped heat release cumulatively at each step. For the $n$th step in the cooling program the trapped heat (i.e. the unrelaxed excess enthalpy) at the beginning of the step is given by

$$
Q_{\mathrm{n}}{ }^{h}=\left(C_{\mathrm{F}} \Delta T_{\mathrm{n}-1}+Q_{\mathrm{n}-1}{ }^{h}\right) \phi\left(t, T_{\mathrm{n}-1}\right) .
$$

The trapped heat consists of two parts. The first term in (8) is the unrelaxed excess enthalpy from the previous $n-1$ th step. The second term is the cumulative unrelaxed excess enthalpy due to all the previous $(n-2)$ steps which remain unrelaxed at the beginning of the $n$th step. [It may be noted that for a property $P$ which follows the relaxation function $\phi(T, t)$ the relaxed part after time $t$ is $P[1-\phi(T, t)]$ and the corresponding unrelaxed part is $P[\phi(T, t)]$. The amount of trapped heat released at 
A model for the analysis of the heat release

the $n$th step is given by

$$
Q_{\mathrm{n}}{ }^{r}=Q_{\mathrm{n}-1}{ }^{h}\left[1-\phi\left(t, T_{\mathrm{n}}\right)\right]
$$

We would like to mention here that incorporating the effect of the delayed heat release is an important aspect of our model. The trapped heat release has a significant effect on the shape of the observed specific heat at the glass transition. The experimentally obtained specific heat cannot be reproduced without including this term: In other words, it is not only the relaxation of the enthalpy at a given temperature step but also the cumulative effect of the unrelaxed enthalpy (which relaxes progressively in subsequent steps) that describes the effective specific heat near the glass transition. The effect of this unrelaxed enthalpy and the associated delayed heat release are incorporated through the terms $Q^{h}$ and $Q^{r}$ in our model.

The temperature dependence of the trapped heat release also shows interesting behavior associated with the glass transition which we discuss in a later section.

From (7) we obtain the cooling rate for the $n$th step as

$$
(\mathrm{d} T / \mathrm{d} t)_{T}=\left(\dot{Q}\left(T_{\mathrm{n}}\right)-\dot{Q}_{\mathrm{n}}\right) / C_{\mathrm{eff}}
$$

where $\dot{Q}\left(T_{n}\right)$ is the rate of heat leak through the link at the temperature $T_{n}$ as determined from the experiment. The heat capacity from the model which is obtained from this cooling rate (as in the actual experiment) can then be calculated from (1). From the total heat capacity, the addenda heat capacity is subtracted to obtain the sample heat capacity.

The parameters which are treated as adjustable in the model are the equilibrium configurational heat capacity $C_{\mathrm{FO}}$ and the parameters associated with the time dependent relaxation time $\tau_{\mathrm{s}}$. These parameters are adjusted to get the best agreement between the specific heat calculated from the model and that obtained from the experiment. The parameters thus obtained are taken to be those representative of the structural relaxation in the system.

\section{Application of the model to the analysis of the heat release in glycerol}

The model described above is now applied to the analysis of the specific heat of glycerol shown in figure 2 . To do so we need to calculate the time dependent $\tau_{\mathrm{s}}$ and the trapped heat release from the cooling program. In this section we discuss our method of obtaining $\tau_{\mathrm{s}}$ as well as the behavior of the trapped heat release $Q^{r}$ and the fictive temperature $T_{\mathrm{F}}$ obtained from the trapped heat $Q^{h}$.

\subsection{The time-dependent relaxation time $\tau_{\mathrm{s}}$}

As mentioned earlier we have adopted Kovacs' method where the nonequilibrium $\tau_{\mathrm{s}}$ is obtained by allowing $\tau_{\mathrm{s}}$ to depend on a quantity like excess enthalpy or excess volume which tracks the deviation of the system from equilibrium. This dependence is introduced by incorporating into the temperature dependence of $\tau_{s}$, a term which retards the increase of $\tau_{\mathrm{s}}$ as temperature is lowered. This term accounts for the effect of high temperature configurations that are frozen-in during cooling. Due to the presence of such non-equilibrium configurations, at any given temperature the system has shorter relaxation times than those associated with equilibrium configurations. 


\section{$M$ Rajeswari and $A K$ Raychaudhuri}

The expression for $\tau_{\mathrm{s}}$ is given by

$$
\tau_{\mathrm{s}}=\tau_{0} \exp [1 /(f+\delta)] \text {. }
$$

The term $\delta$ in (11) has a time dependence given by

$$
\mathrm{d} \delta / \mathrm{d} t=-D q-\left(\delta / \tau_{\mathrm{s}}\right)
$$

In equilibrium $\delta=0$ and (11) should represent the VTF expression in (4). It therefore follows that $f$ has the form

$$
f=\left(T-T_{0}\right) / A
$$

where $A$ and $T_{0}$ correspond to the activation energy and $T_{0}$ as in the VTF expression for $\tau_{\mathrm{s}}$.

In (12) which governs the time dependence of $\delta, q$ is the cooling rate and the parameter $D$ corresponds to the change (across the glass transition) in the derivative of the thermodynamic quantity whose relaxation is being considered. We have treated $D$ as an adjustable parameter in the model. In our experiment we start cooling the liquid from a temperature $T_{\mathrm{A}}>T_{\mathrm{g}}$ after the liquid has been held at $T_{\mathrm{A}}$ long enough to ensure that it attains equilibrium at this temperature. Correspondingly in the model the cooling starts at a temperature where $\delta=0$. The value of $\delta$ at each subsequent temperature step $\Delta T$ is obtained by analytically integrating (12) which gives

$$
\delta(T+\Delta T)=D q \tau_{\mathrm{s}}(T)\left[\exp \left(-\Delta t / \tau_{\mathrm{s}}(T)\right)-1\right]+\delta(T)\left[\exp \left(-\Delta t / \tau_{\mathrm{s}}(T)\right]\right.
$$

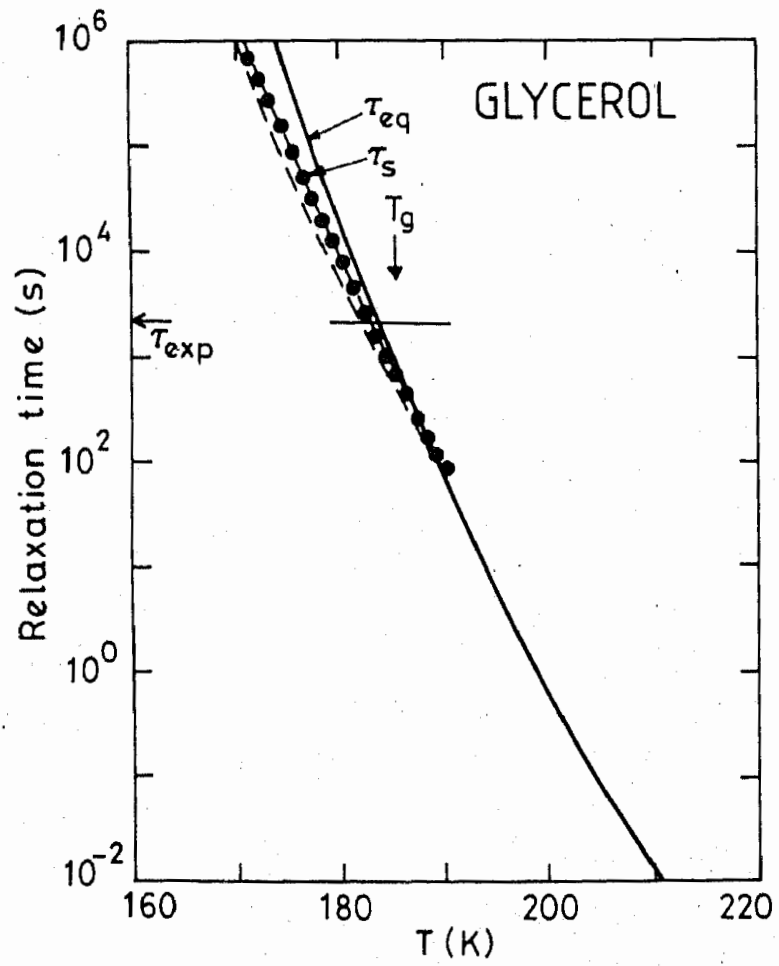

Figure 5. The nonequilibrium relaxation time $\left(\tau_{s}\right)$ as compared to the equilibrium relaxation time $\left(\tau_{\text {eq }}\right)$ and the experimental time scale $\left(\tau_{\text {exp }}\right)$. 
During cooling for each temperature step $\Delta T, \delta$ at the end of the step is calculated from (14) using the value of $\tau_{\mathrm{s}}$ at the beginning of the step. Using this value of $\delta, \tau_{\mathrm{s}}$ at the end of the step is calculated from (1)). This procedure is continued to generate $\tau_{\mathrm{s}}$ at each temperature. The $\tau_{\mathrm{s}}$ thus obtained is used in (1), (8), and (10) to calculate the specific heat from the model. In figure 5 we show the behavior of the time dependent $\tau_{\mathrm{s}}$ for glycerol generated by the above method which is also compared to the experimental time scale $\tau_{\exp }$ and the equilibrium relaxation time $\tau_{\text {eq }}$ calculated from the VTF expression in (4). The values of $\tau_{\mathrm{s}}$ depart from $\tau_{\mathrm{eq}}$ in the glass transition range where $\tau_{\mathrm{s}} \sim \tau_{\text {exp }}$. When $T>T_{\mathrm{g}}$ and $\Delta t \gg \tau_{\mathrm{s}}$ we have $\delta \sim 0$ as a result of which $\tau_{\mathrm{s}} \sim \tau_{\text {eq }}$. When $T \sim T_{\mathrm{g}}, \delta$ becomes large enough to introduce an appreciable difference between $\tau_{\mathrm{s}}$ and $\tau_{\text {eq }}$.

The various parameters in the calculation of $\tau_{\mathbf{s}}$ are discussed in a later section.

\subsection{Temperature dependence of the rate of trapped heat release $Q^{r}$}

Before coming to the results of the analysis we discuss briefly the behavior of the trapped heat release rate $Q^{r}$ in our model. In figure 6(a) we show the temperature dependence of $Q^{r}$ from the model. As seen in the figure. $Q^{r}$ passes through a maximum at $T>T_{\mathrm{g}}$ and falls abruptly at $T \sim T_{\mathrm{g}}$. For glycerol the peak occurs at $\sim 1.03 T_{\mathrm{g}}$. This behavior of $Q^{r}$ may be explained as follows. When $T>T_{\mathrm{g}}, \tau_{\mathrm{s}} \ll \tau_{\exp }$ and the configurational modes equilibrate almost completely in the time scale $\tau_{\text {exp }}$. As a result there is very little trapped heat $Q^{h}$ (excess enthalpy) in the system. As the cooling proceeds, $\tau_{\mathrm{s}}$ increases and the amount of trapped heat $Q^{h}$ as well as the rate of release of the trapped heat $Q^{r}$ increases. As the system is cooled down to $T_{\mathrm{g}}$ and below, though the trapped heat continues to accumulate, the rate of trapped heat release decreases as the condition $\tau_{\mathrm{s}} \gg \tau_{\exp }$ is approached. In other words, the approach to equilibrium has slowed down to such an extent that the trapped heat release is not observable in the experimental time scale $\tau_{\text {exp }}$. This explains the peak at $T_{\mathrm{g}}$. The amount of trapped heat in the system $\left(Q^{h}\right)$ however increases monotonically as the system cools below $T_{\mathrm{g}}$.

\subsection{Fictive temperature from the trapped heat}

Earlier we had briefiy discussed the fictive temperature concept which has been used to calculate the nonequilibrium relaxation times. In this section we discuss how the fictive temperature may be obtained from $Q^{h}$ which we have earlier referred to as the trapped heat in the system (see equation 11). Fictive temperature $T_{\mathrm{F}}$ for enthalpy relaxation is defined by $(10)$

$$
H(T)=H_{\mathrm{e}}\left(T_{\mathrm{F}}\right)+C_{\mathrm{pg}} \mathrm{d} T
$$

where $H_{\mathrm{e}}\left(T_{\mathrm{F}}\right)$ is the equilibrium enthalpy of the liquid at temperature $T_{\mathrm{F}}$ and $C_{\mathrm{pg}}$ is the specific heat of the glass. Equation (15) implies that the configurational enthalpy $H_{\text {conf }}(T)$ corresponds to the equilibrium configurational enthalpy at $T=T_{\mathrm{F}}$.

It follows that the excess enthalpy at $T$ relative to the enthalpy of the equilibrium liquid is given as

$$
H_{\mathrm{ex}}(T)=H_{\mathrm{e}}\left(T_{\mathrm{F}}\right)-H_{\mathrm{e}}(T) \simeq C_{\mathrm{Fo}}\left(T_{\mathrm{F}}-T\right)
$$

(The approximation involved in the last part of this equality is due to a small correction due to the temperature dependence of $C_{\mathrm{Fo}}$ ). 
$M$ Rajeswari and $A$ K Raychaudhuri

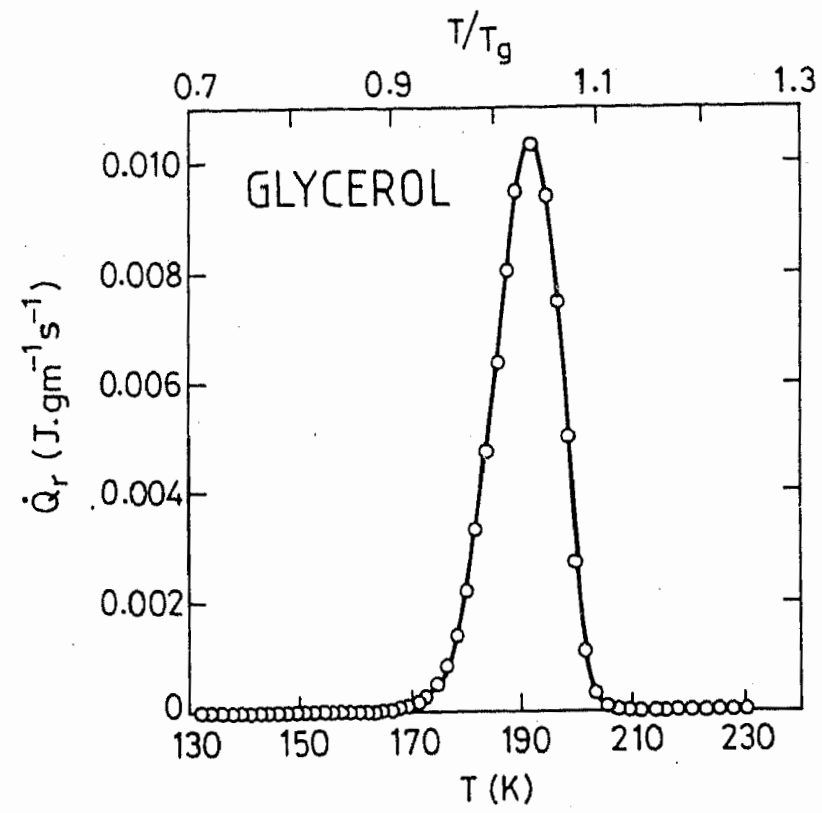

(a)

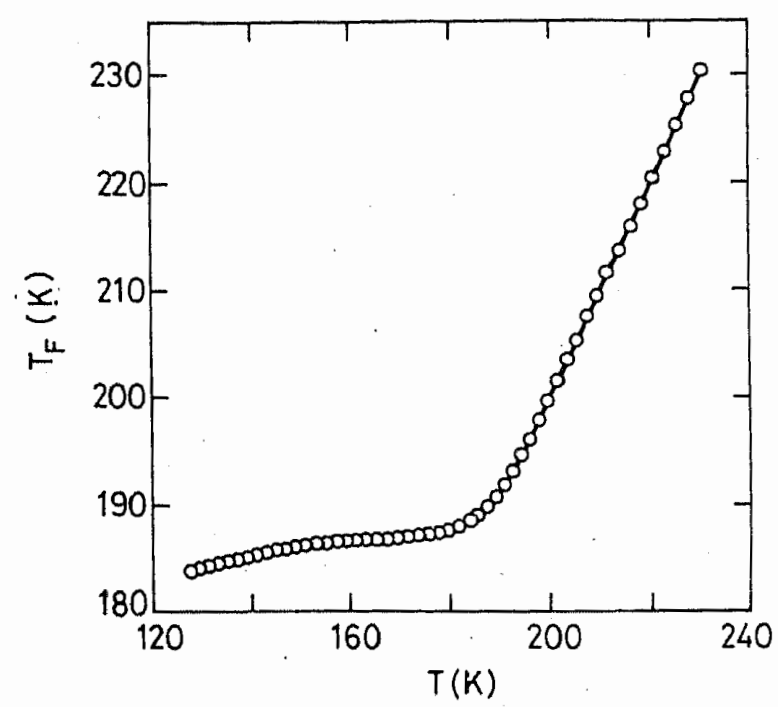

(b)

Figure 6. (a) The rate of trapped heat release as a function of temperature. (b) The fictive temperature $T_{\mathrm{F}}$ calculated from the trapped heat, as a function of temperature.

It can be seen that the excess enthalpy $H_{\text {ex }}$ in (16) is the same as the trapped heat $Q^{h}(T)$ computed in our model according to (8). We thus have

$$
Q^{h}(T)=C_{\mathrm{FO}}\left(T_{\mathrm{F}}-T\right)
$$

We can use (17) to calculate $T_{\mathrm{F}}(T)$ since both $Q^{h}$ and $C_{\mathrm{FO}}$ are obtained from our analysis. The fictive temperature curve thus obtained is shown in figure $6(\mathrm{~b})$. It can be seen that for $T>T_{\mathrm{g}}$ when $\tau_{\mathrm{s}} \ll \tau_{\exp }$ (in the supercooled liquid region), $Q^{h}$ is small and $T_{\mathrm{F}} \sim T$. When $T \sim T_{\mathrm{g}}$, trapped heat is accumulated in the system and $Q^{h}$ builds up to give $T_{\mathrm{F}}>T$. The above discussion of the fictive temperature shows clearly the physical significance of the quantity which we call the trapped heat in our model. 


\section{Results of the analysis for glycerol}

The procedure discussed in the preceding paragraphs has been applied to the analysis of the specific heat data of glycerol shown in figure 2 . The range of the parameters which gives a reasonable agreement between the model and the experiment are identified and these are compared with the corresponding parameters from other experiments. More operational details on the analysis of glycerol as well as the other materials has been reported elsewhere $[9,16]$.

In figure 7 we show the comparison of the specific heat of glycerol calculated from the model with the experimental data. In the inset we show the deviation of the calculated value from the experiment. The model agrees with the experiment within $3 \%$ over a temperature range of $\sim 100 \mathrm{~K}$ spanning the supercooled liquid and glassy regimes. The limit of $3 \%$ is also our experimental precision and accuracy. The crucial point in the quantitative analysis is to find the proper region in the parameter space which gives us the best fit. In addition it is necessary that the parameters are physically justifiable and are in agreement with those obtained from ac specific heat spectroscopy dielectric and mechanical spectroscopy etc. We now discuss the values of the parameters in the model which lead to the best agreement.

\subsection{Lattice heat capacity $C_{L}$}

$C_{\mathrm{L}}$ is taken to be the sum of the heat capacity of crystalline glycerol and that of the addenda. This involves the assumption that the vibrational contribution to the specific heat in the supercooled liquid and glass is the same as that in the crystal which seems to hold within our experimental accuracy of $3 \%$. Both the above quantities are determined from the experiment.

\subsection{Equilibrium configurational heat capacity $C_{F O}$}

This quantity is treated as an adjustable parameter in our model. Our analysis indicates that it is necessary to incorporate a temperature dependence of $C_{\text {FO }}$ to

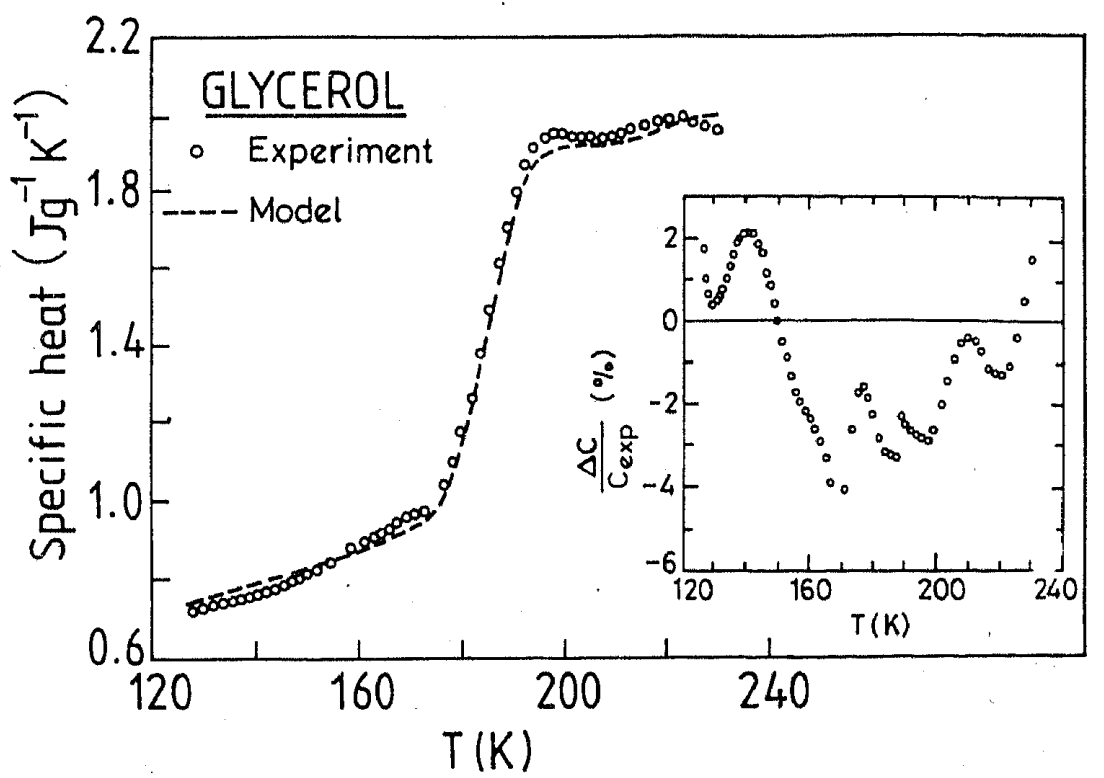

Figure 7. The specific heat of glycerol obtained from the model as compared to the experimental data. The inset shows the \% variation. 


\section{$M$ Rajeswari and $A K$ Raychaudhuri}

obtain the experimentally observed specific heat near the glass transition. $C_{\text {Fo }}$ which gives the best agreement between the model and the experiment is of the form

$$
C_{\mathrm{FO}}(T)=C_{0}+b / T
$$

where $C_{0}$ is taken to be the value of $C_{\text {liq }}-C_{\text {crystal }}$ at $T \sim T_{\mathrm{m}}$ and $b$ is treated as a fit parameter. The increase in the equilibrium configurational specific heat $C_{\mathrm{Fo}}$ according to (22) as seen in our points to the important fact that the drop in the specific heat observed at the glass transition could be attributed entirely to kinetic effects. In other words there is no decrease in the equilibrium configurational specific heat at the glass transition. This is contrary to some of the prevalent ideas which associate the behavior of the specific heat at the glass transition with a phase transition. In figure 8 we compare the behavior of the equilibrium configurational specific heat $C_{\mathrm{FO}}$ with the relaxational (i.e. observable) part of the configurational specific heat given by $C_{\mathrm{FO}}$ $(1-\phi(T, t))$. The relaxational contribution to the observed specific heat becomes comparable to the experimental accuracy at $T<160 \mathrm{~K}$ for glycerol. Hence we can say that the $C_{\mathrm{FO}}(T)$ as seen in our model continues to increase with temperature down to $160 \mathrm{~K}$ i.e. $\left(T / T_{\mathrm{g}} \sim 0.85\right)$. If the rise in $C_{\mathrm{FO}}$ is the signature of the onset of a phase transition it must be occurring below $T / T_{\mathrm{g}} \sim 0.85$. The rise in $C_{\mathrm{Fo}}$ at lower temperatures may also be the tail of a Schottky specific heat associated with the two level systems which must show a peak at a lower temperature. The significant point here is that the temperature dependence of $C_{\text {Fo }}$ at least down to $T / T_{\mathrm{g}} \sim 0.85$ where the relaxational part falls below our experimental accuracy clearly indicates that the observed drop in the specific heat at $T_{\mathrm{g}}$ is purely due to relaxational effects. We consider the indication of a temperature independent $C_{\mathrm{FO}}$ as a significant result of our analysis.

\subsection{Parameters associated with the relaxation time}

These parameters include the activation energy $A$, the pre-exponential factor $\tau_{0}$ in the VTF expression and the parameter $D$ in the calculation of $\delta$ in [12]. The values

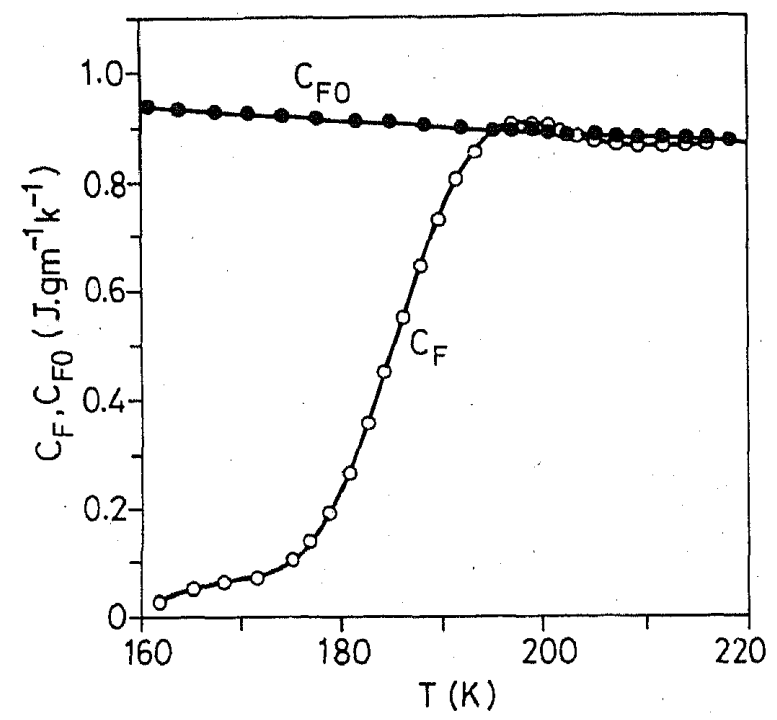

Figure 8. The relaxational part of $C_{\mathrm{FO}}(-\infty)$ compared to the equilibrium configurational specific heat from the model. 
$A$ model for the analysis of the heat release

Table 2. The structural relaxation parameters obtained from the analysis of the continuous cooling calorimetry for glycerol. The numbers in parantheses are those obtained from specific heat spectroscopy.

\begin{tabular}{lll}
\hline & \multicolumn{1}{c}{ Glycerol } & \\
\hline Activation energy & $2500 \pm 100$ & $(2500 \pm 100)^{*}$ \\
$A(\mathrm{~K})$ & $2 \times 10^{-15}-3 \times 10^{-16}$ & $\left(2 \times 10^{-14}-3 \times 10^{-16}\right)^{*}$ \\
$\begin{array}{l}\text { Pre-exponential factor } \\
\tau_{0}(\mathrm{~s})\end{array}$ & $120-128$ & $(128 \pm 5)^{*}$ \\
$\begin{array}{c}\text { Divergence temperature } \\
T_{0}(\mathrm{~K})\end{array}$ & $0.55 \pm 0.02$ & $(0.65 \pm 0.03)$ \\
$\begin{array}{l}\text { Non-debye parameter }(\beta) \\
\text { Parameter } D\left(\mathrm{~K}^{-1}\right) \\
\quad(\text { equation }(16))\end{array}$ & $6 \pm 0.5 \times 10.5$ & \\
$\begin{array}{l}\text { Parameter } b\left(\mathrm{Jg}^{-1}\right) \\
\quad(\text { equation }(19))\end{array}$ & $38-42$ & \\
$\begin{array}{l}\text { Parameter } C_{0}(\mathrm{Jg} \\
\text { (equation }(19))\end{array}$ & $0.68-0.70$ & \\
\hline
\end{tabular}

of these parameters for glycerol are listed in table 2 . Here we briefly comment on these parameters. The value of A obtained from our model is $2500 \pm 100 \mathrm{~K}$, which corresponds to an activation energy of $\sim 10 \mathrm{~kJ} / \mathrm{mol}$. The pre-exponential factor $\tau_{0}$ is in the range of $10^{-15}$ to $10^{-16} \mathrm{~s}$. Both these parameters are.in reasonable agreement with those obtained from specific heat spectroscopy [15]. The parameter $T_{0}$ however shows a different behavior in our analysis. With a constant $T_{0}$ we could not obtain a reasonable agreement between the model and the experiment. The specific heat calculated from the model shown in figure 7 was obtained by smoothly varying $T_{0}$ between $128 \mathrm{~K}$ and $120 \mathrm{~K}$ in the temperature range $190 \mathrm{~K}>T>160 \mathrm{~K}$. A decrease of $T_{0}$ with temperature typically $\sim 7$ to $10 \%$ has also been found for other materials in our study $[9,16]$. The most pronounced decrease of $T_{0}(\sim 24 \%)$ has been seen for the case of propylene carbonate which is also the most fragile (17) system among those we investigated. It has been previously observed that in fragile systems $\tau_{\text {eq }}$ obtained from viscosity measurements reverts back to Arrhenius behavior $\left(T_{0} \approx 0\right)$ as $T \sim T_{\mathrm{g}}$. The decrease of $T_{0}$ observed in our analysis points to a similar behavior. However in systems like glycerol which lie in intermediate range between the strong and fragile glass formers the VTF relation has been found to hold throughout [17]. The tendency of $T_{0}$ for glycerol to decrease as observed in our model is at variance with this earlier idea and questions the universality of the VTF expression for the relaxation time. It should be mentioned that even for propylene carbonate despite the decrease in $T_{0}$ we do not observe a cross-over to complete Arrhenius behavior $\left(T_{0}=0\right)$ down to the lowest temperature limit $\left(T / T_{\mathrm{g}} \sim 0.85\right)$ of our analysis. Similar conclusions have been reached on the basis of ac calorimetry experiments [18] on o-terphenyl mixtures which also represent a fragile system. This in conjunction with our results which seems to suggest that the fragile behavior seen in mechanical relaxation may not be extendible to enthalpy relaxation.

We now come to the parameter $D$ in (16) for the calculation of the quantity $\delta$ which incorporates the time dependence of $\tau_{s}$. In Kovacs' model as applied to volume relaxation [19] $D$ was taken to be the change of the thermal expansion coefficient $(\Delta \alpha)$ at the glass transition. The analogous quantity for enthalpy relaxation should be 


\section{$M$ Rajeswari and $A K$ Raychaudhuri}

represented by $\Delta C_{\mathrm{p}}$ scaled by some appropriate enthalpy. In our model we have treated $D$ as a fit parameter. The values of $D$ which give the best agreement lie in the range $D \sim 6.5 \times 10^{-5} \mathrm{~K}^{-1}$. This value of $D$ corresponds to $D \sim \Delta C_{\mathrm{p}} / H_{0}$ where $H_{0}$ has a magnitude comparable to the zero point enthalpy given by $H_{0} \sim N_{\mathrm{A}} h \omega_{0}$ with $\omega_{0} \sim 2 \pi \tau_{0}$ and $N_{\mathrm{A}}$ is the Avogadro number. For glycerol with $\Delta C_{\mathrm{p}} \sim 100 \mathrm{~J} \mathrm{~mol}^{-1} \mathrm{~K}^{-1}$ we have $D \sim 4 \times 10^{-5} \mathrm{~K}^{-1}$ which is of the order of $D$ obtained from our analysis.

In figure 9 we show the effect of varying $D$ on the nonequilibrium $\tau_{s}$. With increasing values of $D$ (which corresponds to the increasing deviation of the system from equilibrium) $\tau_{\mathrm{s}}$ increasingly departs from the VTF behavior saturating below $T_{\mathrm{g}}$. It is interesting to note the sensitivity of $\tau_{\mathrm{s}}$ to $D$. Below $T_{\mathrm{g}}, \tau_{\mathrm{s}}$ is essentially determined by $\delta$ and hence by the parameter $D$. As $T$ approaches $T_{0}$, the dependence on $D$ prevents the divergence of $\tau_{\mathrm{s}}$ (equations 15 and 17). This also implies that in a real experiment with a finite cooling rate (however small) one cannot see the true divergence of $\tau_{\mathrm{s}}$ because even a small value of $\delta$ will tend to saturate $\tau_{\mathrm{s}}$ as $T$ approaches $T_{0}$.

\subsection{The parameter $\beta$ in the relaxation function}

As mentioned earlier, the parameter $\beta(0<\beta<1)$ in (3) takes into account the non-debye nature of the relaxation function which is related to a spectrum of relaxation times. A value of $\beta=1$ represents Debye relaxation with a single relaxation time, smaller values of $\beta$ corresponding to a broader relaxation time spectrum. For glycerol, our analysis shows that $\beta=0.55 \pm 0.05$ gives a satisfactory agreement between the model and the experiment. We compare this value with that obtained from ac specific heat spectroscopy in the next section.

\subsection{Comparison with ac specific heat spectroscopy}

In table 2 we have given a comparison of the parameters obtained from the analysis of our experiment with those obtained from the specific heat spectroscopy (15). As seen in the table, the activation energy $A$ and the pre-exponential factor $\tau_{0}$ from the

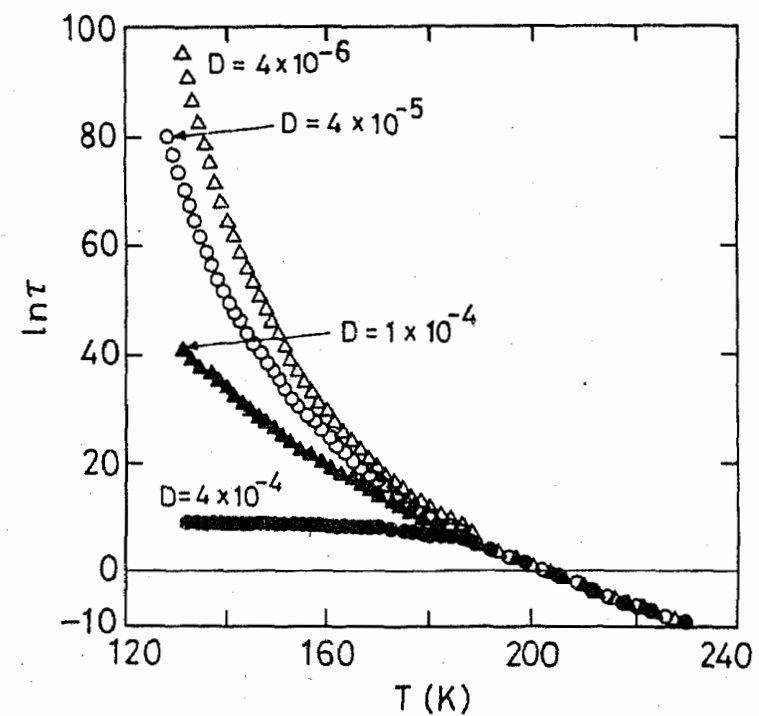

Figure 9. The nonequilibrium $\tau_{\mathbf{s}}$ generated from the different values of $D . D$ : $4 \times 10^{-4}(\mathbf{O}) ; 1 \times 10^{-4}(\Delta) ; 4 \times 10^{-5}(0) ; 4 \times 10^{-6}(\Delta)$. 


\section{A model for the analysis of the heat release}

two experiments are in good agreement. A significant difference is the temperature dependence of the parameter $T_{0}$ in our analysis while $T_{0}$ is temperature independent as obtained from the specific heat spectroscopy experiment. Another important difference is the wider spectrum of relaxation times as indicated by the smaller value of $\beta$ in our experiment. These differences may be understood in view of the following facts. The time scale of the specific heat spectroscopy experiment is much smaller as compared to the present experiment as a result of which the relaxational effects are observable at higher temperatures. In other words, specific heat spectroscopy is probing the equilibrium response at higher temperatures. In contrast, our experiment is most sensitive to these parameters in the glass transition range where the system falls out of equilibrium.

\subsection{The effect of variation of the parameters on the calculated specific heat}

The parameters discussed above almost independently determine the various attributes of the behavior of the calculated specific heat. We observe the following from our analysis:

(1) The equilibrium configurational specific heat $C_{\mathrm{FO}}$ governs the slope of the specific heat in the supercooled liquid state $\left(T>T_{\mathrm{g}}\right)$ close to the glass transition.

(2) The parameters $\tau_{0}, A$ and $T_{0}$ govern the temperature of onset of the glass transition.

(3) The parameter $\beta$ and the temperature dependence of $T_{0}$ affects the slope of the specific heat in the transition range and hence the width of the transition.

The calculated specific heat is most sensitive to these parameters in the transition range as expected since the relaxational contribution is maximum here. A detailed analysis of the effect of each of the parameters on the specific heat in the three regimes - the supercooled liquid region, the glass transition region and the glassy regime - may be found elsewhere [19]. We have been able to deduce these parameters unambiguously within a narrow range of the parameter space by minimizing the difference between the specific heat observed in the experiment and that calculated in the model. An average percentage error $\Delta C$ is defined in the three relaxational regimes mentioned above as

$$
\Delta C=1 / n \Sigma\left|C_{\text {expt }}-C_{\text {model }}\right|
$$

where $n$ is the number of points in each temperature interval over which $\Delta C$ is averaged. We find deep or moderately deep minima in this difference around the best fit values of the parameters. As examples we show in figure $10(a-d)$ the effect of varying the parameters $A, \tau_{0}, D$ and $b$ respectively showing the minimum in $\Delta C$ in a narrow range of the values of these parameters. $A, D$ and $\tau_{0}$ control the relaxation time $\tau_{\mathrm{s}}$ and $b$ governs the temperature dependence of the equilibrium specific heat $C_{\mathrm{FO}}$.

As pointed out earlier, the specific heat in the glass transition interval is most sensitive to $\tau_{\mathrm{s}}$ because in this temperature region the time dependence of the relaxation function $\phi(T, t)$ contributes to the calculated specific heat. At $T \gg T_{\mathrm{g}}, \phi(T, t) \sim 0$ and the system is in thermal equilibrium. At $T \ll T_{\mathrm{g}}, \phi(T, t) \sim 1$ and as a result the variation of $\tau_{\mathrm{s}}$ does not affect the calculated specific heat. If the variation of any of the parameters increase $\tau_{\mathrm{s}}$ then freezing occurs at a higher temperature and for all temperatures $C_{\text {model }}<C_{\text {expt }}$. Similarly, if $\tau_{\mathrm{s}}$ decreases, the glass transition is shifted to lower temperatures and we have $C_{\text {model }}>C_{\text {expt }}$. Of particular interest is the variation in the 

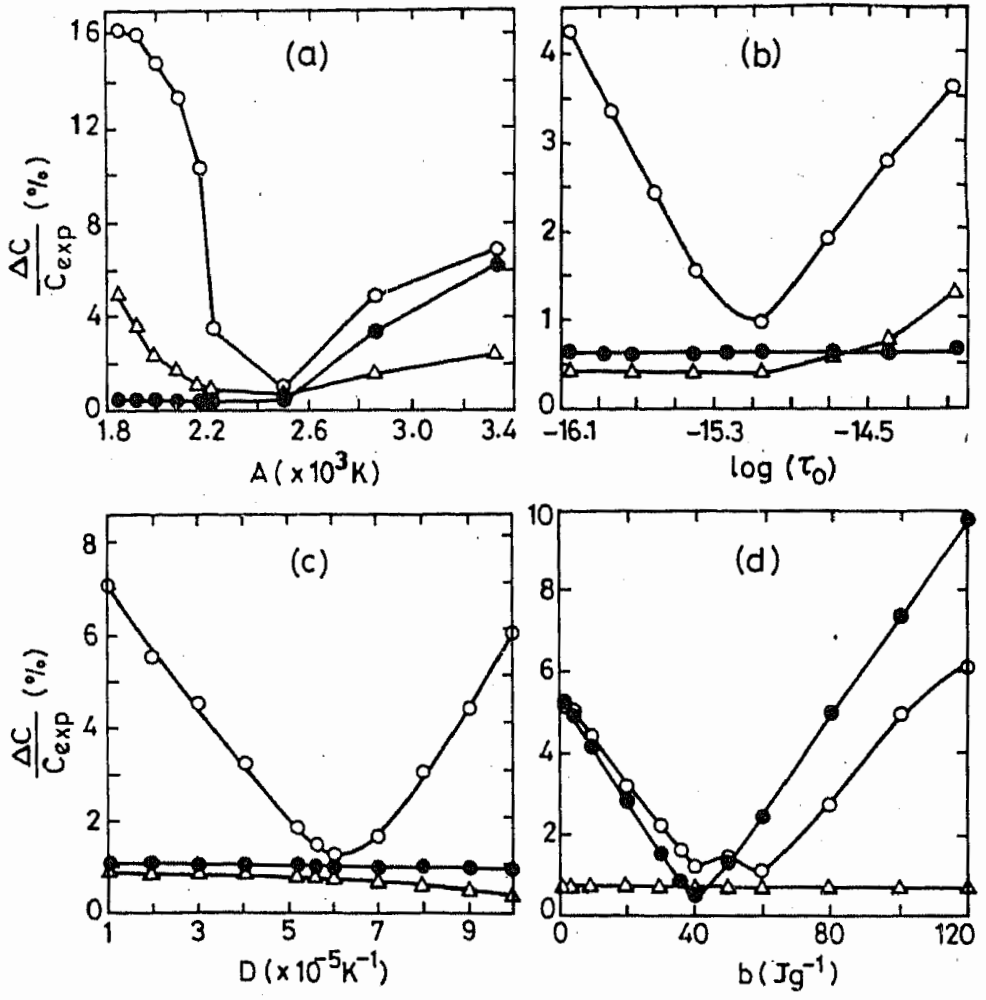

Figure 10a-d. The effect of the parameters $A, D, \tau_{0}$ and $b$ on $\Delta C$ in the three relaxational regimes: supercooled liquid $(\bullet)$; glass transition range $(O)$; sub- $T_{\mathrm{g}}$ region $(\Delta)$. Note that minima are obtained in $\Delta C$ when the parameters have the best fit values.

activation energy $A$, which for a given change around the best fit value $(A \sim 2500 \mathrm{~K})$ gives the maximum change in $\Delta C$ (see figure 10). In the transition range $\Delta C$ is changed almost symmetrically for increasing or decreasing $A$. The situation is somewhat different for the regions in the range $T>T_{\mathrm{g}}$ and $T<T_{\mathrm{g}}$. For the supercooled liquid region ( $T>T_{\mathbf{g}}$ ) since the system is close to thermal equilibrium a decrease in $A$ (i.e. a decrease in $\tau_{\mathrm{s}}$ ) does not lead to any change but an increase in $A$ (i.e. an increase in $\tau_{s}$ leads to the system moving farther out of equilibrium as a result of which $C_{\text {model }}<C_{\text {expt }}$ and $\Delta C$ increases. Similar arguments apply for the sub- $T_{\mathrm{g}}$ region. This method of analysis thus not only helps us to identify the best fit value of the parameter $A$ but also physically reveals the dependence of $C_{\text {model }}$ on the variation of $\tau_{s}$ achieved through the variation of $A$.

The arguments given for $A$ also apply for $D$ and $\tau_{0}$ and one can identify the extent of the change in $C_{\text {model }}$ when these parameters are varied. Since the parameter $b$ controls the slope of $C_{\mathrm{FO}}(T)$ and not $\tau_{\mathrm{s}}$, the variation of $b$ has the largest effect in the supercooled liquid region $\left(T>T_{\mathrm{g}}\right)$ where $C_{\mathrm{Fo}}$ contributes most to the specific heat. As expected the parameter $b$ has no sgnificant effect on the specific heat below the glass transition region where the configuratianal component freezes out.

\section{Concluding summary}

We have presented a model to analyze the specific heat at the glass transition as observed in the continuous cooling calorimetry experiment. In the framework of this model we have been able to obtain several parameters associated with the enthalpy 
A model for the analysis of the heat release

relaxation at the glass transition from the experimentally observed specific heat data. We consider the following as the most significant results of our analysis.

(1) The incorporation of the effects of delayed enthalpy relaxation through the quantity called trapped heat release is an important feature in our model. Our analysis brings out the interesting behavior of this quantity at the glass transition.

(2) The temperature dependence of the equilibrium configurational specific heat points to the fact that the observed fall in the specific heat at the glass transition is entirely relaxational in origin.

(3) The temperature dependence of the parameter $T_{0}$ indicates the tendency of the system to move towards Arrhenius behavior close to the glass transition.

(4) The behavior of the nonequilibrium structural relaxation time and its effect on the observed specific heat is clearly brought out in the model.

(5) The identification of the excess (unrelaxed) enthalpy as the cumulative trapped heat in the model allows us to determine the fictive temperature. The fictive temperature thus obtained shows the expected behavior at the glass transition.

Finally we would like to mention that the model described here has also been applied to the analysis of the specific heat in partially crystallized glasses $[19,20]$ to understand the effects of partial crystallization on the structural relaxation. Further the model is sufficiently general to be applicable to the analysis of any time-domain experiment which probes the structural relaxation at the glass transition. With small modifications, the model may also be extended to include the effects of built-in thermal history where the experiment involves thermal cycling across the glass transition interval.

\section{References}

[1] A K Raychaudhuri and M Rajeswari, Rev. Solid State Sci. 3, (1989) M Rajeswari and A K Raychaudhuri, Euro. Phys. Lett. 10, 699 (1989)

[2] M Rajeswari, S K Ramasesha and A K Raychaudhuri, J. Phys. E21, 101 (1988)

[3] C T Moynihan, A J Easteal, M A deBolt and J Tucker, J. Am. Ceram. Soc. 59, 312 (1976)

[4] G S Parks and H M Huffman, J. Phys. Chem. 31, 1842 (1927)

[5] S S Chang and A B Bestul, J. Chem. Phys. 56, 503 (1974)

[6] N O Birge and S R Nagel, Phys. Rev. Lett. 54, 2674 (1985)

[7] R Bachmann, F J Jr DiSalvo, T H Geballe, R L Greene, R E Howard, C N King, H C Kirsch, K N Lee, R E Schwall, H U Thomas and R B Zubeck, Rev. Sci. Instrum. 43, 205 (1972)

[8] G E Gibson and W F Giaque, J. Am. Chem. Soc. 42, 1547 (1920)

[9] M Rajeswari, 'An experimental study of the specific heat at the glass transition during cooling' Ph D Thesis, Indian Institute of Science, Bangalore (1990)

[10] S A Brawer, J. Chem. Phys. 81, 954 (1984)

[11] C A Angell and W Sichina, Ann. N. Y. Acad. Sci. 279, 53 (1976)

[12] O S Narayanaswami, J. Am. Ceram. Soc. 54, 491 (1971)

[13] A Q Tool, J. Am. Ceram. Soc. 54, 491 (1946)

[14] A J Kovacs, Ann. N. Y. Acad. Sci. 371, 21 (1981)

[15] N O Birge, Phys. Rev. B34, 1631 (1986)

[16] M Rajeswari and A K Raychaudhuri, Phys. Rev. B47, 3036 (1992)

[17] C A Angell, J. Phys. Chem. Sol. 49, 863 (1988)

[18] P Dixon and S R Nagel, Phys. Rev. Lett. 61, 341 (1988)

[19] A Alegria and J Barandiaran, Phys. Status Solidi B120, 349 (1983)

[20] M Rajeswari and A K Raychaudhuri (unpublished) 